\title{
TIGHTER BOUNDS FOR GRAPH STEINER TREE APPROXIMATION*
}

\author{
GABRIEL ROBINS ${ }^{\dagger}$ AND ALEXANDER ZELIKOVSKY ${ }^{\ddagger}$
}

\begin{abstract}
The classical Steiner tree problem in weighted graphs seeks a minimum weight connected subgraph containing a given subset of the vertices (terminals). We present a new polynomialtime heuristic that achieves a best-known approximation ratio of $1+\frac{\ln 3}{2} \approx 1.55$ for general graphs and best-known approximation ratios of $\approx 1.28$ for both quasi-bipartite graphs (i.e., where no two nonterminals are adjacent) and complete graphs with edge weights 1 and 2. Our method is considerably simpler and easier to implement than previous approaches. We also prove the first known nontrivial performance bound (1.5 - OPT) for the iterated 1-Steiner heuristic of Kahng and Robins in quasi-bipartite graphs.
\end{abstract}

Key words. Steiner trees, approximation algorithms, graph Steiner problem, iterated 1-Steiner

AMS subject classifications. 05C05, 05C 85

DOI. $10.1137 /$ S0895480101393155

1. Introduction. Given an arbitrary weighted graph with a distinguished vertex subset, the Steiner tree problem seeks a minimum-cost subtree spanning the distinguished vertices. Steiner trees are important in various applications such as VLSI routing [14], wirelength estimation [7], phylogenetic tree reconstruction in biology [11], and network routing [12]. The Steiner tree problem is $N P$-hard, even in the Euclidean or rectilinear metrics [8], and thus efficient approximation heuristics are sought instead of exact algorithms.

Arora established that Euclidean and rectilinear minimum-cost Steiner trees can be efficiently approximated arbitrarily close to optimal [2]. On the other hand, unless $P=N P$, the Steiner tree problem in general graphs cannot be approximated within a factor of $1+\epsilon$ for sufficiently small $\epsilon>0$ [5]. For arbitrary weighted graphs, the best Steiner approximation ratio achievable within polynomial time was gradually improved from 2 to 1.59 in a series of papers [21, 22, 3, 23, 18, 15, 10].

In this paper we address the graph Steiner tree problem by presenting a polynomialtime approximation scheme with a best-known performance ratio approaching $1+$ $\frac{\ln 3}{2} \approx 1.55$. This improves upon the previously best-known ratio of 1.59 due to Hougardy and Prömel [10]. We apply our heuristic for the Steiner tree problem to quasi-bipartite graphs (i.e., graphs in which no two nonterminals are adjacent), where our heuristic achieves an approximation ratio of $\approx 1.28$ within time $O\left(m n^{2}\right)(m$ and $n$ are the number of terminals and nonterminals in the graph, respectively). This is an improvement over the primal-dual algorithm of Rajagopalan and Vazirani [19], where the bound exceeds 1.5 .

*Received by the editors July 31, 2001; accepted for publication (in revised form) November 3, 2004; published electronically June 16, 2005. A preliminary version of this work appeared in [20]. http://www.siam.org/journals/sidma/19-1/39315.html

$\dagger$ Department of Computer Science, University of Virginia, Charlottesville, VA 22904 (robins@cs. virginia.edu, http://www.cs.virginia.edu/ robins). The work of this author was supported in part by a Packard Foundation Fellowship, by National Science Foundation Young Investigator Award MIP-9457412, and by NSF grant CCR-9988331.

¥Department of Computer Science, Georgia State University, Atlanta, GA 30303 (alexz@cs.gsu. edu, http://www.cs.gsu.edu/ Cscazz). The work of this author was supported in part by NSF grant CCR-9988331 and by a GSU Research Initiation Grant. 
We also show that the well-known iterated 1-Steiner heuristic of Kahng and Robins $[13,9,14]$ achieves an approximation ratio of 1.5 in quasi-bipartite graphs. Previously, no nontrivial bounds were known for the iterated 1-Steiner heuristic. Finally, we improve the approximation ratio achievable for the Steiner tree problem in complete graphs with edge weights 1 and 2 from the previously best-known bound of $\frac{4}{3}$ [5] to less than 1.28 for our algorithm.

The remainder of this paper is organized as follows. In the next section we introduce basic definitions, notation, and properties. In section 3 we present our main algorithm, the k-restricted loss-contracting algorithm ( $k$-LCA $)$. The basic approximation result for the $k$-LCA is proved in section 4 . In sections 5 and 6 we prove an approximation ratio of the $k$-LCA in general graphs and estimate the performance of the iterated 1-Steiner and the $k$-LCA heuristics in both quasi-bipartite graphs and complete graphs with weights 1 and 2 . We conclude in section 7 with possible future research directions.

2. Definitions, notation, and basic properties. Let $G=(V, E, \operatorname{cost})$ be a graph with nonnegative edge costs. Any tree in $G$ spanning a given set of terminals $S \subseteq V$ is called a Steiner tree, and the cost of a tree is defined to be the sum of its edge costs. The Steiner tree problem seeks a minimum-cost Steiner tree for a given terminal set $S$. Any nonterminal vertices contained in a Steiner tree are referred to as Steiner points. We can assume that the graph edge cost function is metric (i.e., the triangle inequality holds) since we can replace any edge $e \in E$ with the shortest path connecting the ends of $e$. Henceforth, we will therefore assume that $G$ is a complete graph. Similarly, for the subgraph $G_{S}$ induced by the terminal set $S$, let $G_{S}$ be the complete graph with vertex set $S$.

Let $M S T\left(G_{S}\right)$ be a minimum spanning tree of $G_{S}$. For any graph $H$, let $\operatorname{cost}(H)$ be the sum of the costs of all edges in $H$. We thus denote the cost of a minimum spanning tree of $H$ by $m s t(H)$, e.g., $\operatorname{cost}\left(M S T\left(G_{S}\right)\right)=m s t\left(G_{S}\right)$. For brevity, we use $m s t$ to denote $m s t\left(G_{S}\right)$.

A Steiner tree over a terminal subset $S^{\prime} \subset S$ in which all terminals $S^{\prime}$ are leaves is called a full component (see Figure 1(a)). Any Steiner tree can be decomposed into full components by splitting all the nonleaf terminals. Our algorithm will proceed by adding full components to a growing solution, based on their "relative cost savings" (this notion will be made precise below). We assume that any full component has its own copy of each Steiner point so that full components chosen by our algorithm do not share Steiner points.

A Steiner tree that does not contain any Steiner points (i.e., where each full component consists of a single edge) will henceforth be called a terminal-spanning tree. Our algorithm will compute relative cost savings with respect to a "shrinking" terminal-spanning tree, which initially coincides with $M S T\left(G_{S}\right)$.

The relative cost saving of a full component is quantified by the ratio of how much that full component decreases the cost of the current terminal-spanning tree over the cost of connecting its Steiner points to terminals. The cost savings of an arbitrary graph $H$ with respect to a terminal-spanning tree $T$ is the difference between the cost of $T$ and the cost of the Steiner tree in the graph obtained by augmenting $H$ with the tree $T$. Let $T[H]$ be the minimum-cost graph in $H \cup T$, which contains $H$ and spans all the terminals of $S$ (see Figure 2). The gain of $H$ with respect to $T$ is defined as $\operatorname{gain}_{T}(H)=\operatorname{cost}(T)-\operatorname{cost}(T[H])$. If $H$ is a Steiner tree, then $\operatorname{gain}_{T}(H)=\operatorname{cost}(T)-\operatorname{cost}(H)$. Note that $\operatorname{gain}_{T}(H) \leq \operatorname{cost}(T)-m s t(T \cup H)$ since $T[H]$ cannot cost less than $M S T(T \cup H)$. In fact, the gain of a full component $K$ 


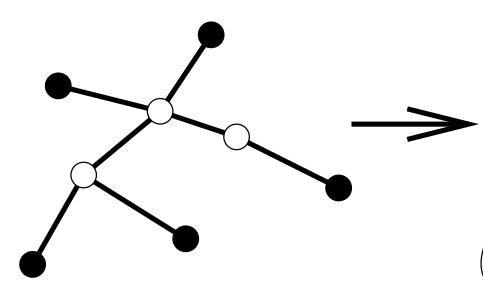

(a)

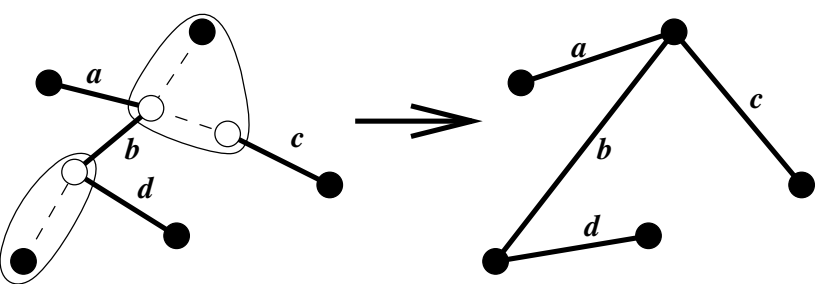

(b)

(c)

FIG. 1. (a) A full component K: filled circles denote terminals and hollow circles denote Steiner points. (b) Connected components of Loss $(K)$ to be collapsed; dashed edges belong to Loss $(K)$. (c) The corresponding terminal-spanning tree $C[K]$ with the contracted Loss $(K)$.

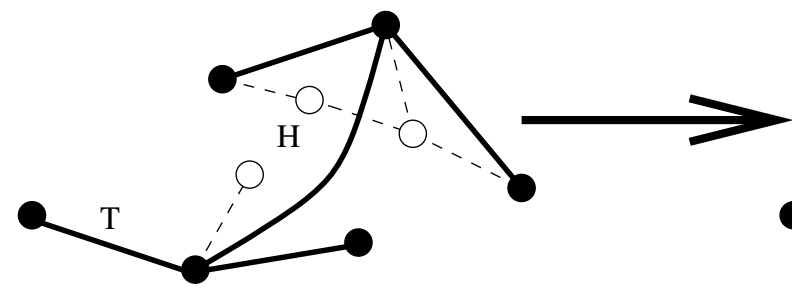

(a)

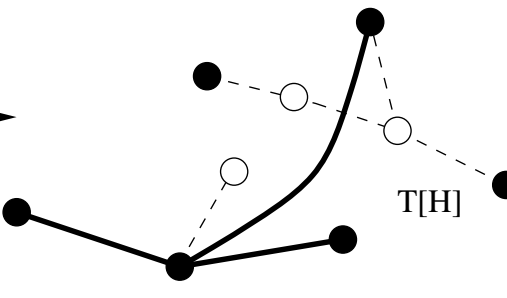

(b)

FIG. 2. (a) A graph $H$ (dashed edges) and a terminal-spanning tree $T$ (solid edges). (b) The corresponding graph $T[H]$ contains $H$ and spans all of the terminals.

also can be defined as

$$
\operatorname{gain}_{T}(K)=\operatorname{cost}(T)-m s t\left(T \cup E_{0}(K)\right)-\operatorname{cost}(K),
$$

where $E_{0}(K)$ are zero-cost edges between all pairs of terminals of $K$. For brevity, the minimum spanning tree of $T \cup E_{0}$ will be referred to as $T / E_{0}$ for any set of zero-cost edges between pairs of terminals in $S$.

We will use the following property of gain (see Lemma 3.3-4, p. 465 in [22] and Lemma 3.14 , p. 391 in [3]). Let $E_{0}$ be an arbitrary set of zero-cost edges between pairs of terminals, and let $K$ be a full component. Then

$$
\operatorname{gain}_{T / E_{0}}(K) \leq \operatorname{gain}_{T}(K) .
$$

This property implies the following key property of gain.

Lemma 2.1. For any terminal-spanning tree $T$ and full components $K_{1}, K_{2}, \ldots, K_{n}$,

$$
\operatorname{gain}_{T}\left(\bigcup_{i=1}^{n} K_{i}\right) \leq \sum_{i=1}^{n} \operatorname{gain}_{T}\left(K_{i}\right) .
$$

Proof. The proof follows from the following chain of inequalities:

$$
\begin{aligned}
\operatorname{gain}_{T}\left(\bigcup_{i=1}^{n} K_{i}\right)= & \operatorname{cost}(T)-\operatorname{cost}\left(T / \bigcup_{i=1}^{n} E_{0}\left(K_{i}\right)\right)-\sum_{i=1}^{n} \operatorname{cost}\left(K_{i}\right) \\
= & \operatorname{cost}(T)-\operatorname{cost}\left(T \cup E_{0}\left(K_{1}\right)\right)-\operatorname{cost}\left(K_{1}\right) \\
& \quad+\operatorname{cost}\left(T / E_{0}\left(K_{1}\right)\right)-\operatorname{cost}\left(T / E_{0}\left(K_{1}\right) \cup E_{0}\left(K_{2}\right)\right)-\operatorname{cost}\left(K_{2}\right)
\end{aligned}
$$




$$
\begin{aligned}
& \quad+\operatorname{cost}\left(T / \bigcup_{i=1}^{n-1} E_{0}\left(K_{i}\right)\right)-\operatorname{cost}\left(T / \bigcup_{i=1}^{n} E_{0}\left(K_{i}\right)\right)-\operatorname{cost}\left(K_{n}\right) \\
= & \sum_{i=1}^{n} \operatorname{gain}_{T / \bigcup_{j \leq i-1} E_{0}\left(K_{j}\right)}\left(K_{i}\right) \\
\leq & \sum_{i=1}^{n} \operatorname{gain}_{T}\left(K_{i}\right) .
\end{aligned}
$$

The minimum-cost connection of the Steiner points of a full component $K$ to its terminals is denoted $\operatorname{Loss}(K)$. Formally, $\operatorname{Loss}(K)$ is a minimum-cost subgraph of $K$ containing a path from each Steiner point of $K$ to one of the terminals of $K$ (see Figure 1(b)). The following lemma gives a simple method of computing $\operatorname{Loss}(K)$.

Lemma 2.2. For any full component $K, \operatorname{Loss}(K)=M S T\left(K \cup E_{0}\right) \backslash E_{0}$, where $K \cup E_{0}$ is $K$ combined with zero-cost edges $E_{0}$ between all pairs of terminals in $K$.

Proof. The forest $F=M S T\left(K \cup E_{0}\right) \backslash E_{0}$ connects all Steiner points of $K$ to the terminals of $K$ and has cost $M S T\left(K \cup E_{0}\right)$. Note that $F$ has the minimum possible cost since $\operatorname{Loss}(K) \cup E_{0}$ spans all the vertices of $K$ and therefore cannot cost more than $M S T\left(K \cup E_{0}\right)$.

Intuitively, Loss will serve as an upper bound on the optimal solution cost increase during our algorithm's execution (as will be elaborated below). We will denote the cost of $\operatorname{Loss}(K)$ by $\operatorname{loss}(K)$. The loss of a union of full components is the sum of their individual losses.

As soon as our algorithm selects a full component $K$ it contracts its $\operatorname{Loss}(K)$, i.e., "collapses" each connected component of $\operatorname{Loss}(K)$ into a single node (see Figure $1(\mathrm{c}))$. Formally, a loss-contracted full component $C[K]$ is a terminal-spanning tree over the terminals of $K$ in which two terminals are connected if there is an edge between the corresponding two connected components in the forest $\operatorname{Loss}(K)$. The cost of any edge in $C[K]$ coincides with the cost of the corresponding edge in $K$. The 1-to-1 correspondence between edges of $K \backslash \operatorname{Loss}(K)$ and $C[K]$ implies that $\operatorname{cost}(H)-\operatorname{loss}(H)=\operatorname{cost}(C[H])$. Similarly, for any Steiner tree $H, C[H]$ is the terminal-spanning tree in which the losses of all full components of $H$ are contracted.

Our algorithm constructs a $k$-restricted Steiner tree, i.e., a Steiner tree in which each full component has at most $k$ terminals. Let $O p t_{k}$ be an optimal $k$-restricted Steiner tree, and let opt $_{k}$ and $\operatorname{loss}_{k}$ be the cost and loss of $O p t_{k}$, respectively. Let opt and loss be the cost and loss of the optimal Steiner tree, respectively.

The following lemma shows that if no $k$-restricted full component can improve a Steiner tree $H$, then $H$ cannot be very expensive; i.e., if we contract the loss of each full component of $H$, then the resulting tree costs no more than an optimal $k$-restricted Steiner tree.

Lemma 2.3. Let $H$ be a Steiner tree; if $\operatorname{gain}_{C[H]}(K) \leq 0$ for any $k$-restricted full component $K$, then

$$
\operatorname{cost}(H)-\operatorname{loss}(H)=\operatorname{cost}(C[H]) \leq \operatorname{opt}_{k} .
$$

Proof. Let $K_{1}, \ldots, K_{p}$ be full components of $O p t_{k}$. The proof follows from the following chain of inequalities:

$$
\begin{aligned}
\operatorname{cost}(C[H])-\text { opt }_{k} & =\operatorname{gain}_{C[H]}\left(O_{p t}\right) \\
& =\operatorname{gain}_{C[H]}\left(K_{1} \cup \cdots \cup K_{p}\right) \\
& \leq \operatorname{gain}_{C[H]}\left(K_{1}\right)+\cdots+\operatorname{gain}_{C[H]}\left(K_{p}\right) \\
& \leq 0 . \quad \square
\end{aligned}
$$


Input: A complete graph $G=(V, E$, cost $)$ with edge costs satisfying the triangle inequality, a set of terminals $S \subseteq V$, and an integer $k, 3 \leq k \leq|S|$

Output: A $k$-restricted Steiner tree in $G$ connecting all the terminals in $S$

$$
\begin{aligned}
& T=M S T\left(G_{S}\right) \\
& H=G_{S} \\
& \text { Repeat forever }
\end{aligned}
$$

Find a $k$-restricted full component $K$ with at least 3 terminals maximizing $r=\operatorname{gain}_{T}(K) / \operatorname{loss}(K)$

If $r \leq 0$ then exit repeat

$H=H \cup K$

$T=M S T(T \cup C[K])$

Output the tree $M S T(H)$

FIG. 3. The $k-L C A$.

An approximation ratio of an algorithm is an upper bound on the ratio of the cost of the found solution over the cost of an optimal solution. In the next section we will propose a new algorithm for the Steiner tree problem and prove a (best-to-date) approximation ratio for it.

3. The algorithm. All previous heuristics (except those of the Berman-Ramaiyer [3] approach) with provably good approximation ratios repeatedly choose appropriate full components and then contract them to form the overall solution. However, this strategy does not allow us to discard an already accepted full component even if later we would find out that a better full component conflicts with a previously accepted component (two components conflict if they share at least two terminals).

The main idea behind the loss-contracting algorithm (see Figure 3) is to contract as little as possible so that (i) a chosen full component may still participate in the overall solution, but (ii) not many other full components would be rejected. In particular, if we contract $\operatorname{Loss}(K)$, i.e., replace a full component $K$ with $C[K]$, then (i) it will not cost anything to add a full component $K$ to the overall solution, and (ii) we decrease the gain of full components, which conflict with $K$ by a small value (e.g., less than in the Berman-Ramaiyer algorithm for large $k$ and much smaller than in [15] for any $k$ ).

Our algorithm iteratively modifies a terminal-spanning tree $T$, which is initially $M S T\left(G_{S}\right)$, by incorporating into $T$ loss-contracted full components greedily chosen from $G$. Each such component $K$ has positive gain, and therefore contains at least three terminals and has nonzero loss. The intuition behind the gain-over-loss objective ratio is as follows. The cost of the approximate solution lies between $m s t=m s t\left(G_{S}\right)$ and $\mathrm{opt}_{k}$. If we accept a component $K$, then it increases (by the gain of $K$ ) the gap between mst and the cost of the approximation. Thus the gain of $K$ is our clear profit. On the other hand, if $K$ does not belong to $O p t_{k}$, then after accepting $K$ we would no longer be able to reach $O p t_{k}$ because we would need to compensate for the connection of incorrectly chosen Steiner points. Therefore, the value of $\operatorname{loss}(K)$, which is the connection cost of Steiner points of $K$ to terminals, is an upper bound on the increase in the cost gap between opt $_{k}$ and the best achievable solution after accepting $K$. Thus $\operatorname{loss}(K)$ is an estimate of our connection expense. Maximizing the ratio of gain over loss is equivalent to maximizing the profit per unit expense.

We now describe a polynomial-time implementation of the $k$-LCA. We first find 
all pairwise distances in the graph $G$. Then, for each $k$-tuple of terminals (there are $|S|^{k}$ of them) it is sufficient to try all possible choices of $k-2$ Steiner points chosen from the nonterminal nodes of $V-S$ because every $k$-restricted full component $K$ is uniquely defined by its Steiner points of degree at least 3. The loss of $K$ can be determined in time $O\left(k^{2}\right)$ by finding the minimum spanning tree of $K \cup E_{0}$ (see Lemma 2.2). Thus, we can find all full Steiner components in time $O\left(|S|^{k} \cdot|V-S|^{k-2}\right)$. Note that the cost and loss do not change in the iterations of the $k$-LCA.

The number of iterations of $k$-LCA cannot exceed the number of full Steiner components $O\left(S^{k}\right)$ since we have $\operatorname{gain}_{T}(K)=0$ after contracting the loss of a full component $K$. The gain of a full component $K$ can be found in time $O(k)$ after precomputing the longest edges between any pair of nodes in the current minimum spanning tree, which may be accomplished in time $O(S \log S)$. Thus, the runtime of all the iterations can be bounded by $O\left(k \cdot S^{2 k+1} \log S\right)$. The total runtime is thus $O\left(|S|^{k} \cdot|V-S|^{k-2}+k \cdot S^{2 k+1} \log S\right)$.

4. Approximation ratio of the $k$-LCA. This section proves the basic approximation result of this paper.

THEOREM 4.1. For any instance of the Steiner tree problem, the cost of the approximate Steiner tree produced by the $k-L C A$ is at most

$$
\text { Approx } \leq \operatorname{loss}_{k} \cdot \ln \left(1+\frac{m s t-o p t_{k}}{\operatorname{loss}_{k}}\right)+\text { opt }_{k} .
$$

Proof. Let $K_{1}, \ldots, K_{\text {last }}$ be full components chosen by the $k$-LCA. Let $T_{0}=$ $M S T\left(G_{S}\right)$ and let $T_{i}, i=1, \ldots$, last be the tree $T$ produced by the $k$-LCA after $i$ iterations. Let $\operatorname{cost}\left(T_{i}\right)$ be the cost of $T_{i}$ after the $i$ th iteration of the $k$-LCA.

LEMMA 4.2. $\operatorname{gain}_{T_{i-1}}\left(K_{i}\right)=\operatorname{cost}\left(T_{i-1}\right)-\operatorname{mst}\left(T_{i-1} \cup K_{i}\right)$.

Proof. It is sufficient to show that $T_{i-1}\left[K_{i}\right]=M S T\left(T_{i-1} \cup K_{i}\right)$. Assume that $M S T\left(T_{i-1} \cup K_{i}\right)$ does not contain some edge $e \in K_{i}$ and let $A$ and $B$ be two connected components of $K_{i}-\{e\}$. We will show that either $A$ or $B$ has a larger gain-over-loss ratio, which contradicts the choice of $K_{i}$.

Since $e$ does not belong to $M S T\left(T_{i-1} \cup K_{i}\right)$, we have $\operatorname{cost}\left(T_{i-1}[A \cup B]\right)<$ $\operatorname{cost}\left(T_{i-1}\left[K_{i}\right]\right)$. By Lemma 2.1, $\operatorname{gain}_{T_{i-1}}\left(K_{i}\right)<\operatorname{gain}_{T_{i-1}}(A \cup B) \leq \operatorname{gain}_{T_{i-1}}(A)+$ $\operatorname{gain}_{T_{i-1}}(B)$. Since $e \notin M S T\left(T_{i-1} \cup K_{i}\right)$, we conclude that $e \notin M S T\left(K_{i} \cup E_{0}\right)$, where $E_{0}$ are zero-cost edges between all terminals of $K_{i}$, and by Lemma 2.2, $e \notin \operatorname{Loss}\left(K_{i}\right)$. Thus $\operatorname{Loss}\left(K_{i}\right)=\operatorname{Loss}(A) \cup \operatorname{Loss}(B)$ and $\operatorname{loss}\left(K_{i}\right)=\operatorname{loss}(A)+\operatorname{loss}(B)$. Finally,

$\frac{\operatorname{gain}_{T_{i-1}}\left(K_{i}\right)}{\operatorname{loss}\left(K_{i}\right)}<\frac{\operatorname{gain}_{T_{i-1}}(A)+\operatorname{gain}_{T_{i-1}}(B)}{\operatorname{loss}(A)+\operatorname{loss}(B)} \leq \max \left\{\frac{\operatorname{gain}_{T_{i-1}}(A)}{\operatorname{loss}(A)}, \frac{\operatorname{gain}_{T_{i-1}}(B)}{\operatorname{loss}(B)}\right\}$.

We define the supergain of a graph $H$ with respect to a Steiner tree $T$ as

$$
\operatorname{supergain}_{T}(H)=\operatorname{gain}_{T}(H)+\operatorname{loss}(H) .
$$

By Lemma 4.2, the supergain of $K_{i}$ with respect to $T_{i-1}$ is

$$
\begin{aligned}
\operatorname{supergain}_{T_{i-1}}\left(K_{i}\right) & =\operatorname{gain}_{T_{i-1}}\left(K_{i}\right)+\operatorname{loss}\left(K_{i}\right) \\
& =\operatorname{cost}\left(T_{i-1}\right)-\operatorname{mst}\left(T_{i-1} \cup K_{i}\right)+\operatorname{mst}\left(T_{i-1} \cup K_{i}\right)-\operatorname{cost}\left(T_{i}\right) \\
& =\operatorname{cost}\left(T_{i-1}\right)-\operatorname{cost}\left(T_{i}\right) .
\end{aligned}
$$

Let $G_{i}=\operatorname{supergain}_{T_{i}}\left(O p t_{k}\right)$ be the supergain of the optimal $k$-restricted Steiner tree $O p t_{k}$ with respect to $T_{i}, i=0,1, \ldots$, last. Let $\operatorname{loss}(n)$ be the loss of the first $n$ 
accepted full trees $K_{1}, \ldots, K_{n}$. We now show that the loss of the full components identified by the $k$-LCA does not grow too fast.

LEMMA 4.3. If $G_{n}$ is positive, then $\frac{\operatorname{loss}(n)}{\operatorname{los} s_{k}} \leq \ln \frac{G_{0}}{G_{n}}$.

Proof. Let $l_{i}=\operatorname{loss}\left(K_{i}\right)$ and $g_{i}=\operatorname{supergain}_{T_{i-1}}\left(K_{i}\right)$ be, respectively, the loss and supergain of the $i$ th full Steiner tree accepted by the $k$-LCA. Let $O p t_{k}$ consist of full components $X_{j}$. By Lemma 2.1,

$$
\begin{aligned}
\frac{G_{0}}{\operatorname{loss}_{k}} \leq \frac{\sum_{X_{j} \in \text { Opt }_{k}} \operatorname{supergain}_{T_{0}}\left(X_{j}\right)}{\sum_{X_{j} \in \text { Opt }_{k}} \operatorname{loss}\left(X_{j}\right)} & \leq 1+\max _{X_{j} \in O p t_{k}}\left\{\frac{\text { gain }_{T_{0}}\left(X_{j}\right)}{\operatorname{loss}\left(X_{j}\right)}\right\} \\
& \leq 1+\frac{\operatorname{gain}_{T_{0}}\left(K_{1}\right)}{\operatorname{loss}\left(K_{1}\right)}=\frac{g_{1}}{l_{1}}
\end{aligned}
$$

Inductively, for $i=1,2, \ldots, n, \frac{G_{i-1}}{\operatorname{loss}_{k}} \leq \frac{g_{i}}{l_{i}}$. Therefore,

$$
g_{i} \geq \frac{l_{i}}{\operatorname{loss}_{k}} G_{i-1} \text {. }
$$

Each time the $k$-LCA accepts a full tree $K_{i}$, it decreases the cost of $T_{i}$ by the supergain of $K_{i}$, which results in a decrease of the supergain of $O p t_{k}$ by the same value. Equality (4.2) yields $G_{i}=\operatorname{cost}\left(T_{i}\right)-\operatorname{cost}\left(O p t_{k}\right)+\operatorname{loss}_{k}$. Therefore, $G_{i-1}-G_{i}=$ $\operatorname{cost}\left(T_{i-1}\right)-\operatorname{cost}\left(T_{i}\right)=g_{i}$.

Inequality (4.3) implies that $G_{i}=G_{i-1}-g_{i} \leq G_{i-1}\left(1-\frac{l_{i}}{l o s s_{k}}\right)$. Since $G_{n}>0$, unraveling the last inequality yields

$$
\frac{G_{n}}{G_{0}} \leq \prod_{i=1}^{n}\left(1-\frac{l_{i}}{\operatorname{loss_{k}}}\right)
$$

Taking the natural logarithms of both sides and using the inequality $x \geq \ln (1+x)$, we finally obtain

$$
\ln \frac{G_{0}}{G_{n}} \geq \sum_{i=1}^{n} \frac{l_{i}}{l o s s_{k}}=\frac{\operatorname{loss}(n)}{\operatorname{los} s_{k}} .
$$

By Lemma 2.3, after the algorithm stops iterating, the cost of the last tree $T_{\text {last }}$ will be at most $\operatorname{opt}_{k}$. We stop iterating when $\operatorname{cost}\left(T_{n+1}\right)<\operatorname{opt}_{k} \leq \operatorname{cost}\left(T_{n}\right)$ for some $n$.

We now show how iteration $n+1$ can be "partially" performed so that $\operatorname{cost}\left(T_{n+1}\right)$ will coincide with opt $t_{k}$. We split $g_{n+1}=\operatorname{supergain}_{T_{n}}\left(K_{n+1}\right)$ into two values $g_{n+1}^{1}$ and $g_{n+1}^{2}$ (i.e., $\left.g_{n+1}=g_{n+1}^{1}+g_{n+1}^{2}\right)$ such that $\operatorname{cost}\left(T_{n}\right)-g_{n+1}^{1}=o p t_{k}$ and, therefore,

$$
\begin{gathered}
g_{n+1}^{1}=\operatorname{cost}\left(T_{n}\right)-o p t_{k} \\
G_{n}-g_{n+1}^{1}=\operatorname{cost}\left(T_{n}\right)-o p t_{k}+\operatorname{loss}_{k}-\left(\operatorname{cost}\left(T_{n}\right)-o p t_{k}\right)=\operatorname{loss}_{k} .
\end{gathered}
$$

We split $l_{n+1}=\operatorname{loss}\left(K_{n+1}\right)$ into $l_{n+1}^{1}$ and $l_{n+1}^{2}$ such that $\frac{g_{n+1}}{l_{n+1}}=\frac{g_{n+1}^{1}}{l_{n+1}^{1}}$. Finally, we set $\operatorname{loss}^{1}(n+1)=\operatorname{loss}(n)+l_{n+1}^{1}$ and

$$
G_{n+1}^{1}=G_{n}-g_{n+1}^{1}>0 .
$$


Since $\frac{g_{n+1}}{l_{n+1}}=\frac{g_{n+1}^{1}}{l_{n+1}^{1}}$, inequality (4.4) implies that

$$
\ln \frac{G_{0}}{G_{n+1}^{1}} \geq \frac{\operatorname{loss}^{1}(n+1)}{\operatorname{loss}_{k}} .
$$

Since $g_{i}=\operatorname{gain}_{T_{i}}\left(K_{i}\right)+\operatorname{loss}\left(K_{i}\right) \geq \operatorname{loss}\left(K_{i}\right)=l_{i}$, we have $\frac{g_{n+1}^{2}}{l_{n+1}^{2}}=\frac{g_{n+1}}{l_{n+1}} \geq 1$, and thus obtain

$$
g_{n+1}^{2} \geq l_{n+1}^{2}
$$

The cost of the approximate Steiner tree after $n+1$ iterations is at most

$$
\begin{aligned}
\operatorname{Approx}(n+1) & =\operatorname{mst}\left(T_{0} \cup K_{1} \cup \cdots \cup K_{n+1}\right) \\
& \leq \operatorname{mst}\left(M S T\left(T_{0} \cup K_{1}\right) \cup K_{2} \cup \cdots \cup K_{n+1}\right)+\operatorname{loss}\left(K_{1}\right) \\
& \leq \operatorname{mst}\left(T_{1} \cup K_{2} \cup \cdots \cup K_{n+1}\right)+\operatorname{loss}\left(K_{1}\right) \\
& \ldots \\
& \leq \operatorname{mst}\left(T_{n} \cup K_{n+1}\right)+\sum_{i=1}^{n} \operatorname{loss}\left(K_{i}\right) \\
& \leq \operatorname{cost}\left(T_{n+1}\right)+\operatorname{loss}(n+1) .
\end{aligned}
$$

Since Approx $(n)$ decreases with $n$, the upper bound on Approx $(n+1)$ also bounds Approx $=$ Approx (last), the output of the $k$-LCA. We complete the proof of inequality (4.1) with the following chain of inequalities:

$$
\begin{aligned}
& \text { Approx } \leq \operatorname{Approx}(n+1) \\
& \leq^{(4.10)} \operatorname{loss}(n+1)+\operatorname{cost}\left(T_{n+1}\right) \\
& =\operatorname{loss}(n)+l_{n+1}^{1}+l_{n+1}^{2}+\operatorname{cost}\left(T_{n}\right)-g_{n+1}^{1}-g_{n+1}^{2} \\
& \leq^{(4.9)} \operatorname{loss}(n)+l_{n+1}^{1}+\operatorname{cost}\left(T_{n}\right)-g_{n+1}^{1} \\
& ={ }^{(4.5)} \operatorname{loss}(n)+l_{n+1}^{1}+o p t_{k} \\
& \leq^{(4.8)} \operatorname{loss}_{k} \cdot \ln \frac{G_{0}}{G_{n+1}^{1}}+\text { opt }_{k} \\
& ={ }^{(4.7)} l o s s_{k} \cdot \ln \frac{m s t-o p t_{k}+\operatorname{loss}_{k}}{G_{n}-g_{n+1}^{1}}+o p t_{k} \\
& { }^{(4.6)} l o s s_{k} \cdot \ln \frac{m s t-o p t_{k}+\operatorname{loss}_{k}}{\operatorname{loss}_{k}}+o p t_{k} \\
& =\quad l o s s_{k} \cdot \ln \left(1+\frac{m s t-o p t_{k}}{l o s s_{k}}\right)+o p t_{k} .
\end{aligned}
$$

5. Performance of the $\boldsymbol{k}$-LCA in general graphs. Our estimate of the performance ratio of the $k$-LCA in arbitrary graphs is based on estimating optimal $k$ restricted Steiner trees. Let $\rho_{k}$ be the worst-case ratio of $\frac{o p t_{k}}{o p t}$. It was shown in [6] that $\rho_{k} \leq 1+\left\lfloor\log _{2} k\right\rfloor^{-1}$. We will show below that the approximation ratio of the $k$-LCA is at most $\rho_{k}\left(1+\frac{1}{2} \ln \left(\frac{4}{\rho_{k}}-1\right)\right)$. Therefore, the approximation ratio of the $k$-LCA converges to $1+\frac{\ln 3}{2}<1.55$ when $k \rightarrow \infty$ since $\lim _{k \rightarrow \infty} \rho_{k}=1$. This is an improvement over the algorithm given by Hougardy and Prömel [10], where the approximation ratio approaches 1.59 . 
THEOREM 5.1. The $k$-LCA has an approximation ratio of at most $\left(1+\frac{1}{2} \ln \left(\frac{4}{\rho_{k}}-\right.\right.$ 1)) $\rho_{k}$.

Proof. Since $m s t \leq 2 \cdot$ opt (see [21]), inequality (4.1) yields the following upper bound on the output tree cost of the $k$-LCA:

$$
\text { Approx } \leq \operatorname{loss}_{k} \cdot \ln \left(1+\frac{2 \cdot o p t-o p t_{k}}{\operatorname{loss}_{k}}\right)+o p t_{k} .
$$

Following [15], we show that for any Steiner tree $T, \operatorname{loss}(T) \leq \frac{1}{2} \operatorname{cost}(T)$. Without loss of generality, we can assume that $T$ is a rooted tree, where all Steiner points have degree at least 3 (degree-2 Steiner points can be disregarded since the graph is complete). For each Steiner point in $T$, choose the shortest outgoing edge; then, all chosen edges (i) connect all Steiner points to terminals (thus having cost of at least $\operatorname{loss}(T)$ ), and (ii) have total cost of at most half the cost of $T$. Therefore

$$
\operatorname{loss}_{k} \leq \frac{1}{2} \text { opt }_{k} .
$$

The partial derivative $\left(\operatorname{loss}_{k} \cdot \ln \left(1+\frac{2 \cdot o p t-o p t_{k}}{\text { loss }_{k}}\right)\right)_{\text {loss }}^{\prime}$ is always positive; the upper bound on Approx is therefore maximized when $\operatorname{loss}_{k}=\frac{1}{2} o p t_{k}$. We thus obtain

$$
\frac{\text { Approx }}{o p t} \leq \frac{o p t_{k}}{o p t} \cdot\left(1+\frac{\ln \left(\frac{4 o p t}{o p t_{k}}-1\right)}{2}\right) .
$$

Since the upper bound above grows when $o p t_{k}$ increases, we can replace $\frac{o p t_{k}}{o p t}$ with the maximum value of $\rho_{k}$.

6. Steiner trees in both quasi-bipartite graphs and complete graphs with edge weights 1 and 2. Recently, Rajagopalan and Vazirani [19] suggested a primal-dual-based algorithm for approximating Steiner trees. They show that their algorithm has an approximation ratio of $1.5+\epsilon$ for quasi-bipartite graphs, i.e., the graphs in which no nonterminals are adjacent. We first show that the well-known iterated 1-Steiner heuristic $[13,9,14]$ has an approximation ratio of 1.5. Next, we apply the $k$-LCA to quasi-bipartite graphs and estimate its runtime. Finally, we prove that the performance ratio of the $k$-LCA for quasi-bipartite graphs is below 1.28.

We also apply the $k$-LCA to the Steiner tree problem in complete graphs with edge weights 1 and 2. Bern and Plassmann [5] proved that this problem is MAX SNP-hard and gave a $\frac{4}{3} \cdot O P T$ approximation algorithm. Applying Lovász's algorithm for the parity matroid problem (see [16]), Berman, Fürer, and Zelikovsky gave a 1.2875-approximation algorithm that was given in [4]. We will show that the performance ratio of the $k$-LCA approaches 1.28 for such graphs, improving on previously achievable bounds.

6.1. The iterated 1-Steiner heuristic. The iterated 1-Steiner heuristic (see $[13,9,14])$ repeatedly adds Steiner points to the terminal set, as long as doing so decreases the cost of the minimum spanning tree over the terminals. Accepted Steiner points are deleted if they become useless, i.e., if their degree becomes 1 or 2 in the minimum spanning tree over the terminals. A generalization of the iterated 1-Steiner heuristic to arbitrary graphs, along with a polynomial-time implementation, is given in [1]. 
Although the iterated 1-Steiner heuristic decreases the minimum spanning tree cost by the maximum possible value at each iteration, we will estimate the cost of the output Steiner tree regardless of how it was obtained. The following theorem will also enable us to estimate the performance ratio of a faster batched variant of the iterated 1-Steiner heuristic $[13,9,14]$.

THEOREM 6.1. Given an instance of the Steiner tree problem in a quasi-bipartite graph $G$, let $H$ be a Steiner tree in $G$ such that (i) any Steiner point has degree at least 3, and (ii) $H$ cannot be improved by adding any other Steiner point, i.e., $m s t(H \cup v) \geq \operatorname{cost}(H)$ for any vertex $v$ in $G$. Then the cost of $H$ is at most 1.5 times the optimal.

Proof. Any full component in quasi-bipartite graphs has only a single Steiner point. Therefore, the loss of any full component equals the cost of the cheapest edge connecting its single Steiner point to a terminal. Since any Steiner point has degree at least 3 (condition (i)), the loss of any full component in $H$ is at most one-third of its cost. Thus, $\operatorname{loss}(H) \leq \frac{1}{3} \cdot \operatorname{cost}(H)$.

We now show that gain $_{C[H]}(K) \leq 0$ for any full component $K$. Condition (ii) implies that $m s t(H \cup K) \geq \operatorname{cost}(H)$. If we contract the loss of $H$, then we can decrease $M S T(H \cup K)$ by at most $\operatorname{loss}(H)$ since reduction by $\operatorname{loss}(H)$ happens only if all edges of $\operatorname{Loss}(H)$ belong to $M S T(H \cup K)$. Therefore, $\operatorname{mst}(C[H] \cup K) \geq \operatorname{mst}(H \cup K)-$ $\operatorname{loss}(H)$ and $m s t(C[H] \cup K) \geq \operatorname{cost}(H)-\operatorname{loss}(H)=\operatorname{cost}(C[H])$. Thus, $\operatorname{gain}_{C[H]}(K) \leq$ $\operatorname{cost}(C[H])-m s t(C[H] \cup K) \leq 0$. By Lemma $2.3, \operatorname{cost}(H)-\operatorname{loss}(H) \leq o p t$, and since loss $(H) \leq \frac{1}{3} \cdot \operatorname{cost}(H)$, we obtain $\operatorname{cost}(H) \leq \frac{3}{2} \cdot$ opt.

The above result helps to explain why the iterated 1-Steiner and RajagopalanVazirani heuristics perform similarly when applied to instances of the Steiner tree problem restricted to the rectilinear plane (see [17]).

6.2. Runtime of the $\boldsymbol{k}$-LCA in quasi-bipartite graphs. For a given Steiner point $v$, the $k$-LCA adds only a full component with the largest gain, since the loss is determined by $v$. We can find a full tree with maximum gain with respect to a terminal-spanning tree $T$, among all possible full components with Steiner point $v$, by merely finding all neighbors of $v$ in $M S T(T \cup v)$. Therefore, a full component maximizing the gain-over-loss ratio over all $k$ can be found within polynomial time.

We estimate the runtime of the $k$-LCA for quasi-bipartite graphs as follows. Let $m$ and $n$ be the number of terminals and nonterminals, respectively. The number of iterations is $O(n)$ since a Steiner point can be added only once into $H$. Each iteration consists of $O(n)$ gain evaluations, each of which can be computed within $O(m)$ time. Using the appropriate data structures, the $k$-LCA can be implemented within a total runtime of $O\left(n^{2} \cdot m\right)$, where $m$ is the number of terminals and $n$ is the number of nonterminals.

6.3. Performance bound of the $\boldsymbol{k}$-LCA for special graphs. We first estimate the loss of a Steiner tree in quasi-bipartite graphs and in complete graphs with edge weights 1 and 2 .

Lemma 6.2. For the Steiner tree problem in quasi-bipartite graphs and in complete graphs with edge weights 1 and 2,

$$
m s t \leq 2\left(\text { opt }_{k}-\operatorname{loss}_{k}\right) \text {. }
$$

Proof. For quasi-bipartite graphs, let $K$ be an arbitrary full component of a Steiner tree $T$ with $p$ terminals connected by a single Steiner point with edges of lengths $d_{0}, d_{1}, \ldots, d_{p-1}$. Assume that $\operatorname{loss}(K)=d_{0}=\min \left\{d_{i}\right\}$. Let $m s t(K)$ be the 
cost of a minimum spanning tree of $G_{S^{\prime}}$, where $S^{\prime}$ is the set of terminals in $K$. By the triangle inequality, we have

$$
m s t(K) \leq \sum_{i=1}^{p-1}\left(d_{0}+d_{i}\right)=p \cdot d_{0}+\operatorname{cost}(K)-2 d_{0} \leq 2 \operatorname{cost}(K)-2 \operatorname{loss}(K) .
$$

The bound (6.1) follows from the fact that mst, the cost of a minimum spanning tree over $S$, does not exceed the sum of mst-costs for terminals in each of the full components in $O p t_{k}$.

Now we prove the lemma for the case of complete graphs with edge weights 1 and 2. Let $m$ and $n$, respectively, be the number of terminals and Steiner points in the optimal $k$-restricted Steiner tree $O p t_{k}$. Then $m s t \leq 2 m-2$ since all edge weights are at most 2, and $\operatorname{opt}_{k} \geq m+n-1$ since $O p t_{k}$ contains $m+n$ nodes. We may assume that full components of $O p t_{k}$ contain only edges of weight 1, and therefore $l o s s_{k}=n$. Thus, $m s t \leq 2 m-2=2(m+n-1-n) \leq 2\left(\right.$ opt $_{k}-$ loss $\left._{k}\right)$.

THEOREM 6.3. The $k-L C A$ has an approximation ratio of at most $\approx 1.279$ for quasi-bipartite graphs and an approximation ratio approaching $\approx 1.279$ for complete graphs with edge weights 1 and 2.

Proof. After substituting the minimum spanning tree bound (6.1) into inequality (4.1), we obtain

$$
\text { Approx } \leq \operatorname{loss}_{k} \cdot \ln \left(\frac{o p t_{k}}{\operatorname{loss}_{k}}-1\right)+o p t_{k}
$$

Taking the partial derivative of $\left(\text { loss } \cdot \ln \left(\frac{o p t_{k}}{\text { loss }_{k}}-1\right)\right)_{\text {loss }_{k}}^{\prime}$, we see that the single maximum of the upper bound (6.2) occurs when $x=\frac{\text { loss }_{k}}{\text { opt }_{k}-\operatorname{loss}_{k}}$ is the root of the equation $1+\ln x+x=0$. Solving this equation numerically, we obtain $x \approx 0.279$. Finally, we substitute $x$ into (6.2), yielding

$$
\text { Approx } \leq \frac{x}{1+x} \cdot \text { opt }_{k} \cdot \ln \frac{1}{x}+\text { opt }_{k}=(x+1) \cdot \text { opt }_{k} \approx 1.279 \cdot \text { opt }_{k} .
$$

The bound above is valid for the output of the $k$-LCA for quasi-bipartite graphs if we set $k=|S|$, i.e., if we omit the index $k$. For complete graphs with edge weights 1 and 2, opt $_{k}$ converges to opt, and the approximation ratio of the $k$-LCA therefore converges to 1.279 when $k \rightarrow \infty$.

7. Conclusions and open problems. We presented a new best-performing polynomial-time heuristic for the classical graph Steiner tree problem. This heuristic, called the $k$-restricted loss-contracting algorithm ( $k$-LCA), can be applied to arbitrary metric spaces. The worst-case performance for the $k$-LCA depends on the Steiner ratio and the loss of the optimal Steiner tree (i.e., the cost of connecting Steiner points to terminals). We proved that the $k$-LCA is currently the best approximation heuristic for the Steiner tree problem in graphs: its approximation ratio is $\approx 1.55$ for general graphs and $\approx 1.28$ for both quasi-bipartite graphs and graphs with edge costs 1 and 2. We also used our techniques to derive the first known nontrivial performance ratio $(1.5 \cdot \mathrm{OPT})$ for the iterated 1-Steiner heuristic of Kahng and Robins [13, 9, 14, 1] in quasi-bipartite graphs.

Chief among the remaining open problems is finding heuristics for the classical graph Steiner problem with improved performance bounds. Other special cases of the Steiner problem for special metrics, cost functions, and graph types may be explored 
separately, where it may be possible to exploit the specific underlying structure to further improve the performance bounds. Interestingly, our $k$-LCA is the first (and so far the only) heuristic that is proven to work well for all of the special graph types discussed above.

From a practical perspective, for any given fixed performance bound it would be useful to minimize the running times of the associated heuristics and to quantify and explore various tradeoffs between running times and solution quality. Finally, it would be useful to implement the various heuristics and explore their practical runtime and empirical solution quality by comparing the performance of these implementations side by side on various classes and sizes of inputs.

Acknowledgments. We thank Gruia Călinescu for reading earlier drafts of this paper and giving numerous helpful suggestions.

\section{REFERENCES}

[1] M. J. Alexander And G. Robins, New performance-driven FPGA routing algorithms, IEEE Trans. Comput. Aided Design Integrated Circuits and Systems, 15 (1996), pp. 1505-1517.

[2] S. Arora, Polynomial time approximation schemes for Euclidean TSP and other geometric problems, J. ACM, 45 (1998), pp. 753-782.

[3] P. Berman and V. Ramaiyer, Improved approximations for the Steiner tree problem, J. Algorithms, 17 (1994), pp. 381-408.

[4] P. Berman, M. Fürer, and A. Zelikovsky, Applications of the Matroid Parity Problem to Approximating Steiner Trees, Tech. report 980021, Computer Science Department, UCLA, Los Angeles, 1998. Available online at ftp://ftp.cs.ucla.edu/tech-report/1998-reports.

[5] M. Bern and P. Plassmann, The Steiner tree problem with edge lengths 1 and 2, Inform. Process. Lett., 32 (1989), pp. 171-176.

[6] A. Borchers And D.-Z. Du, The k-Steiner ratio in graphs, SIAM J. Comput., 26 (1997), pp. 857-869.

[7] A. Caldwell, A. B. Kahng, S. Mantik, I. Markov, and A. Zelikovsky, On wirelength estimations for row-based placement, in Proceedings of the International Symposium on Physical Design, ACM, New York, 1998, pp. 4-11.

[8] M. R. Garey And D. S. Johnson, The rectilinear Steiner tree problem is NP-complete, SIAM J. Appl. Math., 32 (1977), pp. 826-834.

[9] J. Griffith, G. Robins, J. S. Salowe, and T. Zhang, Closing the gap: Near-optimal Steiner trees in polynomial time, IEEE Trans. Comput. Aided Design Integrated Circuits and Systems, 13 (1994), pp. 1351-1365.

[10] S. Hougardy, And H. J. Prömel, A 1.598 approximation algorithm for the Steiner problem in graphs, in Proceedings of the Tenth Annual ACM-SIAM Symposium on Discrete Algorithms, SIAM, Philadelphia, ACM, New York, 1999, pp. 448-453.

[11] F. K. Hwang, D. S. Richards, and P. Winter, The Steiner Tree Problem, North-Holland, Amsterdam, 1992.

[12] B. Korte, H. J. Prömel, And A. Steger, Steiner trees in VLSI-layout, in Paths, Flows, and VLSI-Layout, B. Korte et al., eds., Springer, Berlin, 1990, pp. 185-214.

[13] A. B. Kahng And G. Robins, A new class of iterative Steiner tree heuristics with good performance, IEEE Trans. Comput. Aided Design Integrated Circuits and Systems, 11 (1992), pp. 893-902.

[14] A. B. Kahng and G. Robins, On Optimal Interconnections for VLSI, Kluwer, Dordrecht, 1995.

[15] M. Karpinski and A. Zelikovsky, New approximation algorithms for the Steiner tree problem, J. Combin. Optim., 1 (1997), pp. 47-65.

[16] L. Lovász And M. D. Plummer, Matching Theory, Elsevier Science, Amsterdam, 1986.

[17] I. I. Mandoiu, V. V. Vazirani, and J. L. Ganley, A new heuristic for rectilinear Steiner trees, IEEE Trans. Comput. Aided Design Integrated Circuits and Systems, 19 (2000), pp. 1129-1139.

[18] H. J. Prömel And A. Steger, RNC-approximation algorithms for the Steiner problem, in Proceedings of the 14th Annual Symposium on Theoretical Aspects of Computer Science, Springer, Berlin, 1997, pp. 559-570. 
[19] S. Rajagopalan and V. V. Vazirani, On the bidirected cut relaxation for the metric Steiner tree problem, in Proceedings of Annual ACM-SIAM Symposium on Discrete Algorithms, SIAM, Philadelphia, ACM, New York, 1999, pp. 742-751.

[20] G. Robins And A. Zelikovsky, Improved Steiner tree approximation in graphs, in Proceedings of the 11th Annual ACM-SIAM Symposium on Discrete Algorithms, SIAM, Philadelphia, ACM, New York, 2000, pp. 770-779.

[21] H. TAkahashi and A. Matsuyama, An approximate solution for the Steiner problem in graphs, Math. Jap., 24 (1980), pp. 573-577.

[22] A. Zelikovsky, An 11/6-approximation algorithm for the network Steiner problem, Algorithmica, 9 (1993), pp. 463-470.

[23] A. Zelikovsky, Better Approximation Bounds for the Network and Euclidean Steiner Tree Problems, Tech. report CS-96-06, University of Virginia, Charlottesville, VA, 1996. 